ARRHYTHMIAS AND PACING

\section{The year in cardiology: arrhythmias and pacing The year in cardiology 2019}

A. John Camm*, Gregory Y.H. Lip ${ }^{\#, \dagger}$, Richard Schilling", Hugh Calkins ${ }^{\rfloor}$and Jan Steffel ${ }^{\infty}$

*St George's University of London, Cardiology Clinical Academic Group, Molecular \& Clinical Sciences Institute, London,

United Kingdom

"Liverpool Centre for Cardiovascular Science, University of

Liverpool, Liverpool Heart \& Chest Hospital, Liverpool,

United Kingdom

${ }^{\dagger}$ Aalborg Thrombosis Research Unit, Department of Clinical

Medicine, Aalborg University, Aalborg, Denmark

鄚arts Heart Centre, London, United Kingdom

Johns Hopkins Medical Institutions, Baltimore, Maryland,

United States of America

`Department of Cardiology, Division of Electrophysiology,

University Hospital Zurich, Switzerland

Address for correspondence:

Prof A. John Camm

St George's University of London

Cardiology Clinical Academic Group

Molecular \& Clinical Sciences Institute

CranmerTerrace

Tooting

London SWI7 ORE

United Kingdom

Email:

jcamm@sgul.ac.uk

\section{INTRODUCTION}

During this last year, there has been much progress with regard to anticoagulant and ablation therapy for atrial fibrillation (AF). Apart from recently issued European Society of Cardiology Guidelines for the management of patients with supraventricular arrhythmias, there has been little progress in research in this field. Ventricular arrhythmias and device therapy have seen modest progress.

\section{SUPRAVENTRICULAR TACHYCARDIAS}

This year has seen several publications on the ECG diagnosis of supraventricular tachycardia (SVT) $)^{(1-4)}$ and interest in new consumer-led discovery of supraventricular arrhythmias. ${ }^{(5)}$ EP mapping technology has provided better mapping of SVT. ${ }^{(6)}$ There has been a surprising interest in new antiarrhythmic drugs for SVT, ranging for intranasal etripamil (an L-type calcium antagonist) for termination of SVT ${ }^{(7,8)}$ and nifekalant to increase the refractoriness of accessory pathways and reduce the rate of pre-excited supraventricular arrhythmias. ${ }^{(9)}$

\section{Guidelines}

2019 saw new European Society of Cardiology guidelines for the management of patients with $\mathrm{SVT} \mathrm{T}^{(10)}$ which had previously been updated in 2003. However, there was little that was very new. The guidelines insisted that ablation was the best initial management for most re-entrant atrial and $\mathrm{AV}$ junctional tachycardia. However, atrial tachycardia occurring after ablation for AF should not be considered for ablation until at least 3 months after the AF ablation procedure. The guidelines stressed that ablation for AV nodal re-entrant tachycardia could be achieved in almost all without risk of AV block. An invasive EP risk assessment of Wolff-Parkinson-White syndrome was recommended, even in patients who are asymptomatic but have high-risk occupations or are competitive athletes. The guidelines recommend ablation in high risk or symptomatic WPW patients, but stop short of recommending ablation of all accessory pathways. It is pointed out that SVT may cause tachycardia-mediated cardiomyopathy and that ablation may not only eliminate the tachycardia but restore ventricular function.

There are strong Class III recommendations - "what not to do", mostly related to antiarrhythmic drug therapy (Figure I).

\section{ATRIAL FIBRILLATION RISK ASSESSMENT AND TREATMENT DECISIONS}

Various studies have highlighted new developments in the risk assessment for the development of AF and its complications, as well as the use of the non-vitamin $\mathrm{K}$ antagonist oral anticoagulants (NOACs) as thromboprophylaxis.

\section{Risk assessment}

Numerous clinical factors associated with incident AF have been described, (II) but a simple, practical and reliable approach to identifying patients at risk of incident $A F$ is needed.

Clinical factors such as change in body mass index have been associated with an increased risk of $A F^{(12)}$ as has disordered sleep pattern. ${ }^{(13)}$ Various clinical risk scores for identifying incident AF have been described, and as with most clinical 
scores, all have modest predictive value for identifying high-risk patients, and, until recently, there have been complex models derived from multivariate analyses. The C2HEST score was derived and validated in Asia and has recently been externally validated in a French post-stroke cohort and the Danish nationwide registries. ${ }^{(14,15)}$ This would facilitate targeted intensive screening for $A F$, for example, in the post-stroke population with $A F$, where oral anticoagulation $(O A C)$ as secondary prevention is well established. In contrast, two randomised trials in embolic stroke of unknown source (ESUS) using NOACs failed to show a significant reduction in recurrent stroke, while one trial (NAVIGATE-ESUS) showed an excess of bleeds. ${ }^{(16,17)}$

Screening for AF has attracted much attention, with population-based approaches and new technologies.(18) The Apple Watch study investigated if a smartwatch-based irregular pulse notification algorithm identified possible AF, and reported that among participants who received notification of an irregular pulse, $34 \%$ had atrial fibrillation AF on subsequent ECG patch readings and $84 \%$ of notifications were concordant with AF.(19) The Huawei Heart Study also showed the usefulness of photoplethysmographic (PPG)-based technology in population screening for $\mathrm{AF}$, with the positive predictive value of
PPG signals being $91.6 \%$ and leading to improved anticoagulation use $(>80 \%) .{ }^{(20)}$

Risk assessment continues to evolve, with availability of new data showing stroke risks associated with AF patients with hypertrophic cardiomyopathy ${ }^{(21)}$ and imaging-documented significant coronary artery lesions. ${ }^{(22)}$ There has been much interest in the use of sophisticated methods such as machinelearning, even predicting incident AF from a simple 12-lead ECG. ${ }^{(23)}$ More complex risk assessment approaches improve AF stroke risk prediction (at least statistically), but need to be balanced against simplicity and practical application. For now, an independent Patient Cantered Outcome Research Institute (PCORI)-sponsored systematic review and evidence appraisal identified that among the commonly used risk stratification schemes in patients with $A F$, the $C_{2} A_{2} D S_{2} V A S c$ and HAS-BLED scores were the best predictors for stroke and bleeding risks, respectively. ${ }^{(24)}$ Bleeding risk prediction only focused on modifiable bleeding risk factors is an inferior strategy to a formal risk assessment using the HAS-BLED score. ${ }^{(25,26)}$

Stroke and bleeding risk assessments incorporating biomarkers have been proposed based on highly selected anticoagulated

\begin{tabular}{|c|c|c|}
\hline Verapamil is not recommended in wide QRS-complex tachycardia of unknown aetiology. & III & B \\
\hline \multicolumn{3}{|l|}{ Recommendations for the therapy of MRATs } \\
\hline \multicolumn{3}{|l|}{ Acute therapy } \\
\hline Propafenone and flecainide are not recommended for conversion to sinus rhythm. & III & B \\
\hline \multicolumn{3}{|l|}{ Recommendations for the therapy of AVRT due to manifest or concealed APs } \\
\hline \multicolumn{3}{|l|}{ Chronic therapy } \\
\hline $\begin{array}{l}\text { Digoxin, beta-blockers, diltiazem, verapamil and amiodarone are not recommended and are potentially harmful in patients with } \\
\text { pre-excited AF. }\end{array}$ & III & B \\
\hline \multicolumn{3}{|l|}{ Recommendations for the acute therapy of pre-excited AF } \\
\hline \multicolumn{3}{|l|}{ Haemodynamically stable patients } \\
\hline Amiodarone (i.v.) is not recommended. & III & B \\
\hline \multicolumn{3}{|l|}{ Recommendations for the therapy of SVTs in congenital heart disease in adults } \\
\hline \multicolumn{3}{|l|}{ Chronic therapy } \\
\hline Sotalol is not recommended as a first-line antiarrhythmic drug as it is related to an increased risk of pro-arrhythmias and mortality. & III & C \\
\hline $\begin{array}{l}\text { Flecainide and propafenone are not recommended as firstline antiarrhythmic drugs in patients with ventricular dysfunction and } \\
\text { severe fibrosis. }\end{array}$ & III & C \\
\hline
\end{tabular}

FIGURE I: Some "What not to do" recommendations from the 2019 ESC Guidelines on the management of patients with supraventricular tachycardia. 
clinical trial cohorts, but "real-world" studies have not shown the usefulness of such schemes. One study showing sequential addition of biomarkers did not improve the usefulness of stroke and bleeding risk prediction. (27) Also, there are no data across the patient pathway, when first diagnosed and non-anticoagulated, or on aspirin - and following the initiation of OAC. Of note, many risk factors are based on baseline risk assessment, but do not remain static and change with age and incident risk factors. ${ }^{(25,28)}$ Thus, AF assessment is not a "one off" item and needs to be reassessed at regular intervals, e.g. every 4 - 6 months. ${ }^{(29)}$

Non-vitamin $\mathrm{K}$ antagonist oral anticoagulants and atrial fibrillation management in clinical practice

The NOACs have changed the landscape of stroke prevention in AF. These drugs are now the preferred OAC option in most guidelines, but challenges remain in their use among high-risk subgroups that were under-represented in clinical trials, as well as adherence and persistence.

Clinical trial cohorts are selected populations and may be at lower risk compared to "real-world" clinical practice data. ${ }^{(30)}$ The year also saw the first publications of real-world data for edoxaban, which was the fourth NOAC to enter the market. ${ }^{(31)}$ Increasing data for the NOACs in the elderly have been published, ${ }^{(32,33)}$ clearly showing their effectiveness and safety even in very elderly subjects, aged $\geq 80$. Additional data emphasise the importance of using the appropriate labeladherent dosing to ensure best outcomes, as well as persistence data with the NOACs, for example, with dabigatran. ${ }^{(34)}$ One trial, AEGEAN, showed high adherence and persistence with apixaban ( 90\%), but did not show additional benefit from interventions to improve adherence/ persistence. ${ }^{(35)}$

Also, studies of NOAC use in extremes of renal function, both severe renal impairment and supra-normal renal function are important. The latter is pertinent given that all 3 Factor $\mathrm{Xa}_{\mathrm{a}}$ inhibitors showed numerically more ischaemic strokes in the subgroup with $\mathrm{CrCl}>95 \mathrm{~mL} / \mathrm{min}$ when compared with warfarin in their pivotal trials, although this is not apparent in real-world observational data.(36) In end-stage renal failure, observational data show better safety for apixaban over warfarin. ${ }^{(37)}$

The last year has seen new trials with NOACs in catheter ablation (CA) for $A F$, and in the setting of $A F$ patients presenting with an ACS or undergoing PCl/stenting. For $\mathrm{CA}$, an uninterrupted NOAC-based strategy appears to be a safer option compared to a warfarin-based strategy. ${ }^{(38-40)}$ In AF/ACS/PCI patients, the publication to AUGUSTUS and ENTRUST-AF PCI completes the trials of NOACs in this clinical setting. ${ }^{(41,42)}$ These trials suggest that when OAC is used, a NOAC-based regime or a dual therapy (i.e. OAC plus a $\mathrm{P} 2 \mathrm{Y} I 2$ inhibitor) is associated with less major bleeding. ${ }^{(43)}$ Of the overall thrombotic or ischaemic outcomes, there is little difference between a triple therapy or dual therapy approach, or a NOAC-based strategy compared to a warfarin-based strategy. However, a dual therapy approach may be associated with an excess of stent thrombosis and myocardial ischaemic events, and thus patients who are at high risk of such outcomes may merit a short period of triple therapy at the start. In stable coronary disease, OAC alone is associated with better outcomes compared to dual therapy, in the AFIRE trial.(44)

While the concept of integrated AF management has been proposed, its application and implementation in a simple, userfriendly manner have not been previously validated. Integrated care has been associated with reduced mortality and hospitalisation. ${ }^{(46)}$ One integrated and holistic approach to AF management, streamlining the decision-making management approaches that would be uniformly applicable across the whole AF patient pathway, starting with primary care and linking with secondary care (including cardiologist/non-cardiologists), and understandable for the AF patients per se, is the ABC (Atrial fibrillation Better Care) pathway: Avoid stroke; Better symptom management with patient-centred symptom directed decisions on rate or rhythm control; and Cardiovascular and risk factor optimisation, including lifestyle changes $^{(45)}$ (Figure 2). The ABC pathway approach has now been shown in independent studies to be associated with a reduction in mortality, hospitalisation and adverse outcomes, as well as reduced healthcare costs, when compared to "non$A B C$ " adherent management. ${ }^{(47-50)}$ The $A B C$ pathway was tested in a cluster randomised trial showing improved clinical outcomes with an ABC pathway management based on an interactive App that included risk assessments, patient decision aids, educational materials and dynamic tracking of risk (mAFAII trial; ${ }^{(20)}$ presented as Late Breaking Science at the ESC congress, September 2019).

\section{ABLATION}

\section{Clinical outcomes}

A number of publications have described AF CA outcomes and impact on prognosis. Probably the most eagerly awaited was the CABANA study. ${ }^{(51)}$ This multicentre study randomised 2204 patients to CA or drug therapy. As designed, intention to treat, the study was neutral for CA impacting on the primary composite endpoint of death, disabling stroke, serious bleeding, or cardiac arrest. This type of study is incredibly difficult to recruit for because the clinicians most likely to recruit are seeing a patient referred for a CA, so even if they are prepared to enter the study, the cross-over rate is likely to be high from drug to ablation, as it was in this study (27.5\%). When analysing by treatment, there was a prognostic benefit, but this subverts the principle of randomisation and increases bias. 


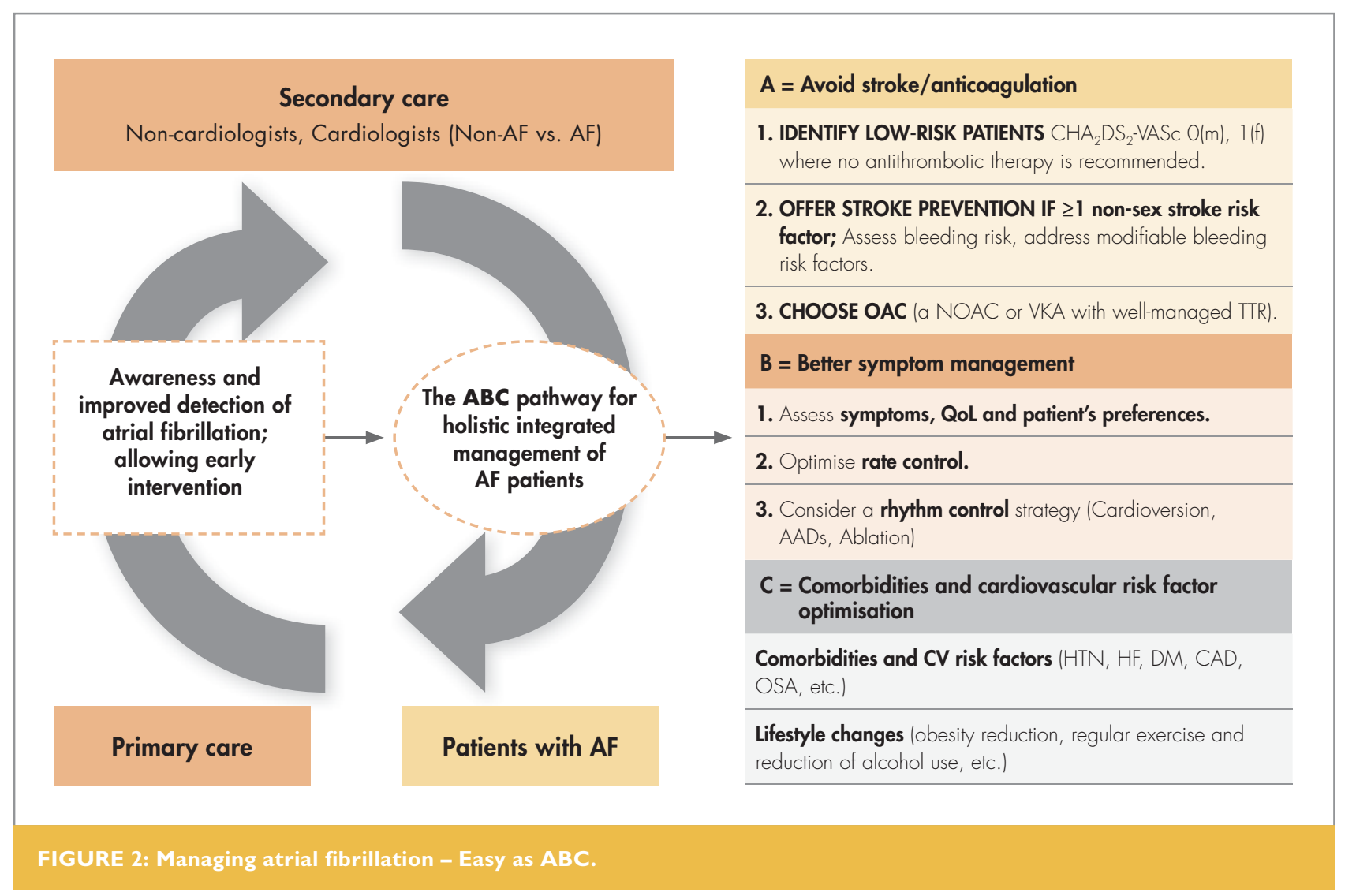

The cerebral micro-emboli associated with AF CA do not appear to have much impact and CA itself may improve cognitive impairment as in the 308 patients studied and followed for I year. ${ }^{(52)}$

Most electrophysiologists continue to tell patients that the primary goal of AF ablation is quality of life (QOL). The first randomised controlled trials (RCT) of $A F C A$ vs. drugs to examine $\mathrm{QOL}$ as the primary endpoint were published in 2019 and favoured CA. ${ }^{(53)}$ While this was a small study, with 155 patients, it does open the way for double-blind RCTs of AF CA with QOL as the primary outcome.

The use of cryoablation for AF has accumulated more evidence this year: it is faster than RF CA, ${ }^{(54)}$ associated with lower risk of pericardial effusion, ${ }^{(55,56)}$ and has superior outcomes ${ }^{(54,55)}$ regardless of centre volume. ${ }^{(57)}$

Several large registries have published this year. The Swedish registry reveals $C A$ procedure complications and death were low and that $A F$, ventricular tachycardia $(\mathrm{VT})$, and premature ventricular complex (PVC) CA numbers increased, with AF having the highest repeat procedure rate (4I\%). ${ }^{(58)}$ A European registry demonstrated that cryoablation is as effective for female patients, but is associated with higher complication rates. ${ }^{(59)}$ The Danish registry confirmed that success rates for
AFL ablation were $90 \%$, but that $\mathrm{AF}$ is a common presentation (13\%) within the 2 years after. ${ }^{(60)}$ The German Helios registry showed that pericardial effusion rates were $0.9 \%$ in 21 I 4 I AF $\mathrm{CA}$, and were more likely in low volume centres, but only if RF was used rather than cryo. ${ }^{(55)}$

CA of VF storm after myocardial infarction was reported in a multicentre study of 110 patients. ${ }^{(61)}$ In-hospital mortality (27\%) and 2-year follow-up mortality (36\%) were high and associated with the time taken to perform CA.

A retrospective study of 110 patients demonstrated $C A$ of recurrent $V T$ in patients with arrhythmogenic ventricular cardiomyopathy, is no more effective than drugs, but is more likely to be successful if both epicardial and endocardial approaches are used. ${ }^{(62)}$

\section{New mapping technologies}

It is recognised that the primary reasons for failure of CA in complex arrhythmia are a lack of understanding of the mechanism. There continues to be huge effort to solve this. This year ripple mapping has been used successfully in persistent AF ( 8 months $53 \%$ vs. $39 \%$ conventional), ${ }^{(64)}$ atrial tachycardia, ${ }^{(65)}$ and $V T$ in arrhythmogenic right ventricular cardiomyopathy (ARVC). ${ }^{(66)}$ Non-contact mapping is returning to clinical practice with an observational trial showed good outcomes for persis- 
tent AF CA at 12 months (59\%). ${ }^{(67)}$ The STAR mapping system (Figure 3) presented its feasibility clinical trial of 35 patients showing freedom from AF after persistent AF CA guided by STAR of $80 \%$ at 18 months. ${ }^{\left({ }^{(68)}\right)}$ It remains to be seen whether any of these make it to widespread clinical use.

\section{Energy sources}

High power short-duration RF may make point-by-point AF CA faster and, at least so far, not being associated with worse outcomes. ${ }^{(63)}$ Electroporation is also showing promise as a novel energy source that is highly effective with low complication rates. ${ }^{(69)}$ The use of radiotherapy to treat intractable VT is an exciting innovation, showing promising results in a small prospective study of 19 patients. ${ }^{(70)}$

\section{Guidelines and consensus statements}

A number of guidelines have been published this year and while these are useful reviews of the literature, the temptation to accept them as dogma has to be resisted, given that they are often drive by consensus of a well-intentioned writing group rather than hard data. The CA of ventricular arrhythmia (VA) guideline suggests that programmed electrical stimulation may come back into fashion as a method for prognostic prediction, this time in patients with frequent PVCs and structural heart disease, and also recommends use of ICE for VA abla- tion, although much of the world does not use ICE without any apparent compromise to their outcomes. ${ }^{(71)}$ The sex differences in arrhythmia consensus highlighted that although outcomes may be different, this should not influence provision of CA for females. ${ }^{(72)}$

\section{VENTRICULAR ARRHYTHMIAS}

Arrhythmogenic cardiomyopathy

This has been an exciting year in arrhythmogenic cardiomyopathy (ACM). There are major publications to be aware of. The first is the Heart Rhythm Society Consensus Document on Arrhythmogenic Cardiomyopathy. ${ }^{(73)}$ This document, which was led by McKenna and Towbin, redefines ACM as a condition that presents with symptomatic and/or asymptomatic arrhythmias in association with some degree of cardiac dysfunction. This "big tent" approach includes classic ARVC, the more recently described arrhythmogenic left ventricular cardiomyopathy, as well as other subgroups of patients. Included within ACM are sarcoidosis, Chagas disease, myocarditis, and a large number of inherited cardiomyopathies. This is a comprehensive and provocative article that is important to be aware of. One of the writing group's goals was to encourage having patients present with arrhythmias and a cardiomyopathy to a specialised centre that performs comprehensive

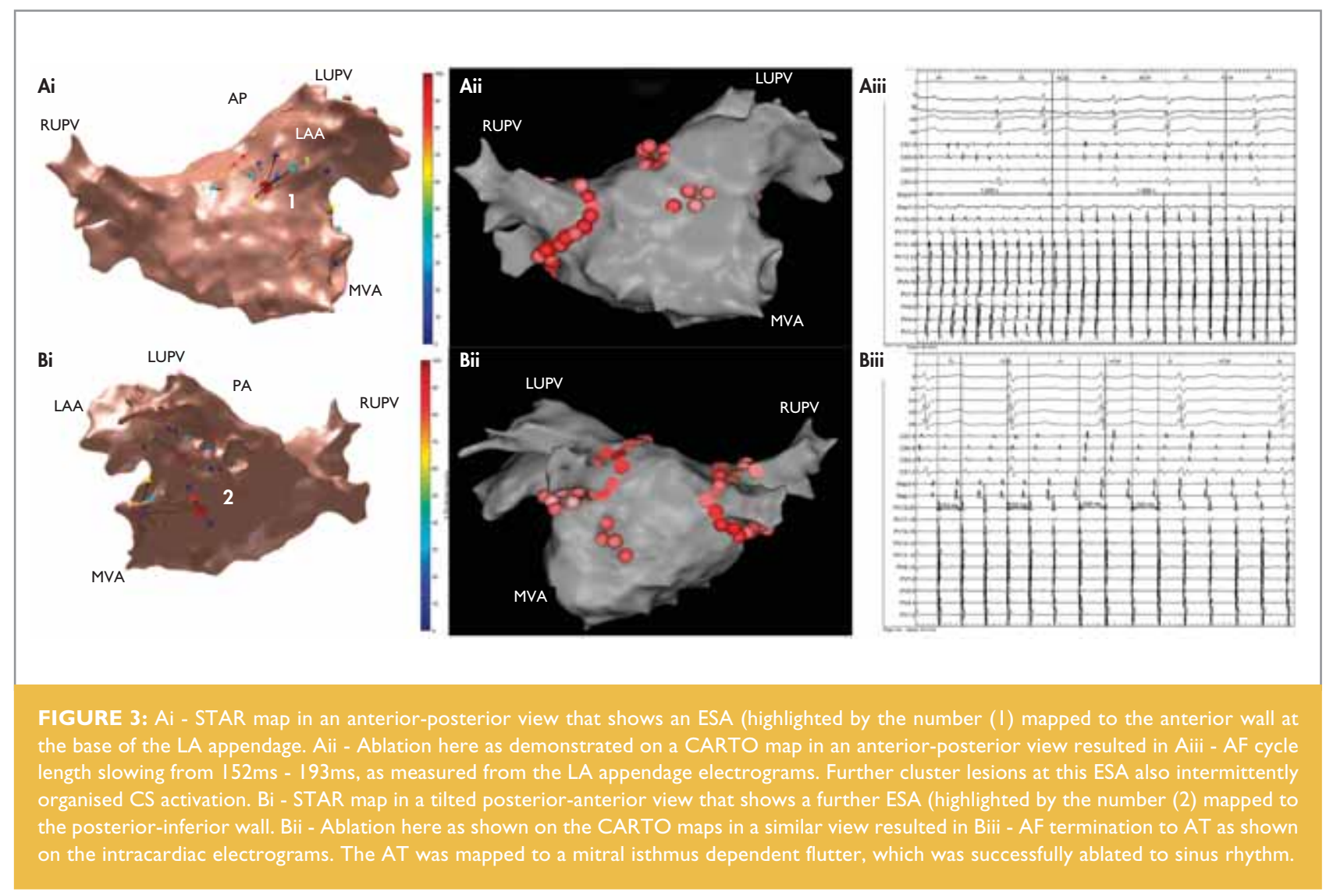


evaluation, arranges for genetic testing, and determines a patient's arrhythmic risk and need for an ICD. ${ }^{(74)}$

Another important publication was authored by CadrinTourigny, et al. ${ }^{(74)}$ Through the combined efforts of 5 international ARVC registries, an ARVC risk calculator was developed to help estimate arrhythmic risk and inform decisions regarding ICD implantation (www.ARVCrisk.com). More than 500 ARVC patients from 5 registries in North America and Europe were enrolled. During 5 years of follow-up, 28\% experienced sustained $V T$, sudden death, or received an appropriate ICD therapy. A prediction model to estimate annual arrhythmic risk was developed (Figure 4). The variables at baseline included in the model are recent syncope, age, gender, non-sustained VT, the number of PVCs in 24 hours, and right ventricular ejection fraction. Furthermore, a final paper by Chatterjee, et al. ${ }^{(75)}$ investigated the diagnostic value of an anti-Desmoglein-2 antibody in diagnosing ARVC. An antibody to DSG-2 was identified in 12/12 and 25/25 ARVC cohorts and $7 / 8$ borderline subjects. The antibody was absent in 11/12 and 20/20 control cohorts. The authors concluded that anti-DSG-2 antibodies are a sensitive and specific marker for ARVC. Before this test can be used clinically, it will need to be tested in more controlled populations, including those with cardiac sarcoidosis.

\section{Cardiac arrest}

Sondergaard, et al. ${ }^{(76)}$ examined the use of bystander CPR among patients who experienced out of hospital cardiac arrest in Denmark. More than three-fourths of cardiac arrests occurred in residential locations. Bystander CPR increased between 2001 and 2004 from 36\% - 84\% in public locations and from $16 \%-61 \%$ in residential locations. Not surprisingly, the increased use of CPR resulted in an increased 30-day survival from $6 \%$ - $25 \%$ for arrests in public locations and from $3 \%-10 \%$ in residential locations.

\section{CARDIAC DEVICES}

What is the evidence behind current guideline recommendations for primary prevention ICD implantation in our present day and age? Can patient populations, background therapies and treatment algorithms, particular in heart failure, and underlying trials conducted well over a decade ago be extrapolated to current daily clinical practice? (Figure 5). ${ }^{(77)}$ According to a large analysis from the French-British-Swedish-Czech CRT Network, death due to progressive heart failure remains the leading cause of death for most patients. ${ }^{(78)}$ Moreover, increasing evidence indicates left ventricular (LV) remodelling as a main driver or arrhythmogenic events leading to sudden cardiac death (SCD), which may be reduced by modalities aimed at preventing (or even reversing) these processes, i.e. neurohormonal blockade and cardiac resynchronisation therapy

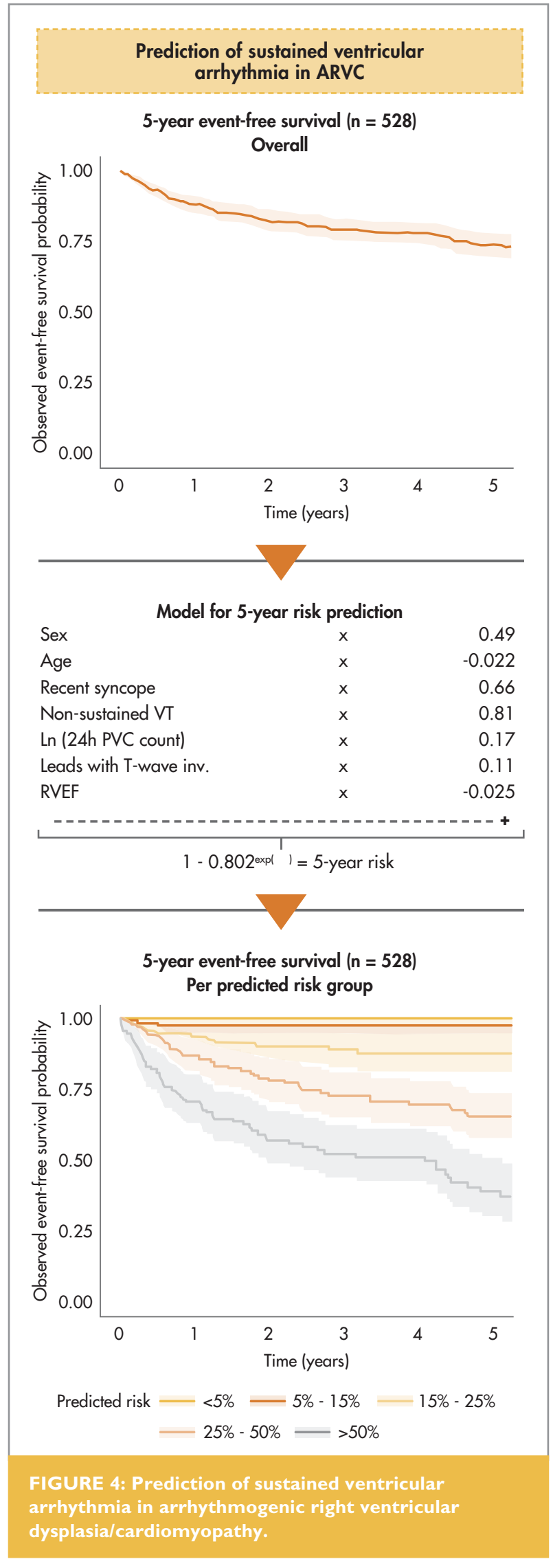

ARVC = arrhythmogenic right ventricular dysplasia/cardiomyopathy, inv. = inversion, $P V C=$ premature ventricular complex, RVEF = right ventricular ejection fraction, VT $=$ ventricular tachycardia. ${ }^{(74)}$ 
(CRT). ${ }^{(79)}$ These concepts and findings call into question the validity of the available randomised clinical trial evidence underlying current recommendations for primary prevention ICD implantation in heart failure patients. On a conceptual level, they additionally raise the question if trials should generally come with a "due date", after which they would require re-validation. On the flipside, however, device therapies have advanced over the last decades, including better algorithms to detect ventricular arrhythmias and to prevent inadequate shocks, as well as the development of extravascular systems such as the S-ICD and the extravascular (EV-) ICD. ${ }^{\left({ }^{80}\right)}$ Indeed, even entirely leadless CRT systems appear to be feasible. ${ }^{(81)}$ If proven safe and effective in the (ongoing) large RCTs, these novel modalities will come with a substantially reduced system-related morbidity, which may again tip the scale towards device-based SCD prevention. Indeed, inadequate shocks, as well as infections, remain the most devastating complications of current ICD systems, which come along with a substantial impact on quality of life, morbidity, and mortality. ${ }^{(82)}$

In addition, better means of risk prediction for SCD above and beyond left ventricular ejection fraction (LVEF) are desperately needed in order to better protect those patients who need it (and prevent those who do not from unnecessary device implantation). One such risk prediction model for patients post-myocardial infarction with preserved LVEF has recently been put forward using electrocardiographic non-invasive risk factors (PVCs, non-sustained VT, late potentials, prolonged QTc, increased T-wave alternans, reduced heart rate variability, and abnormal deceleration capacity with abnormal turbulence),

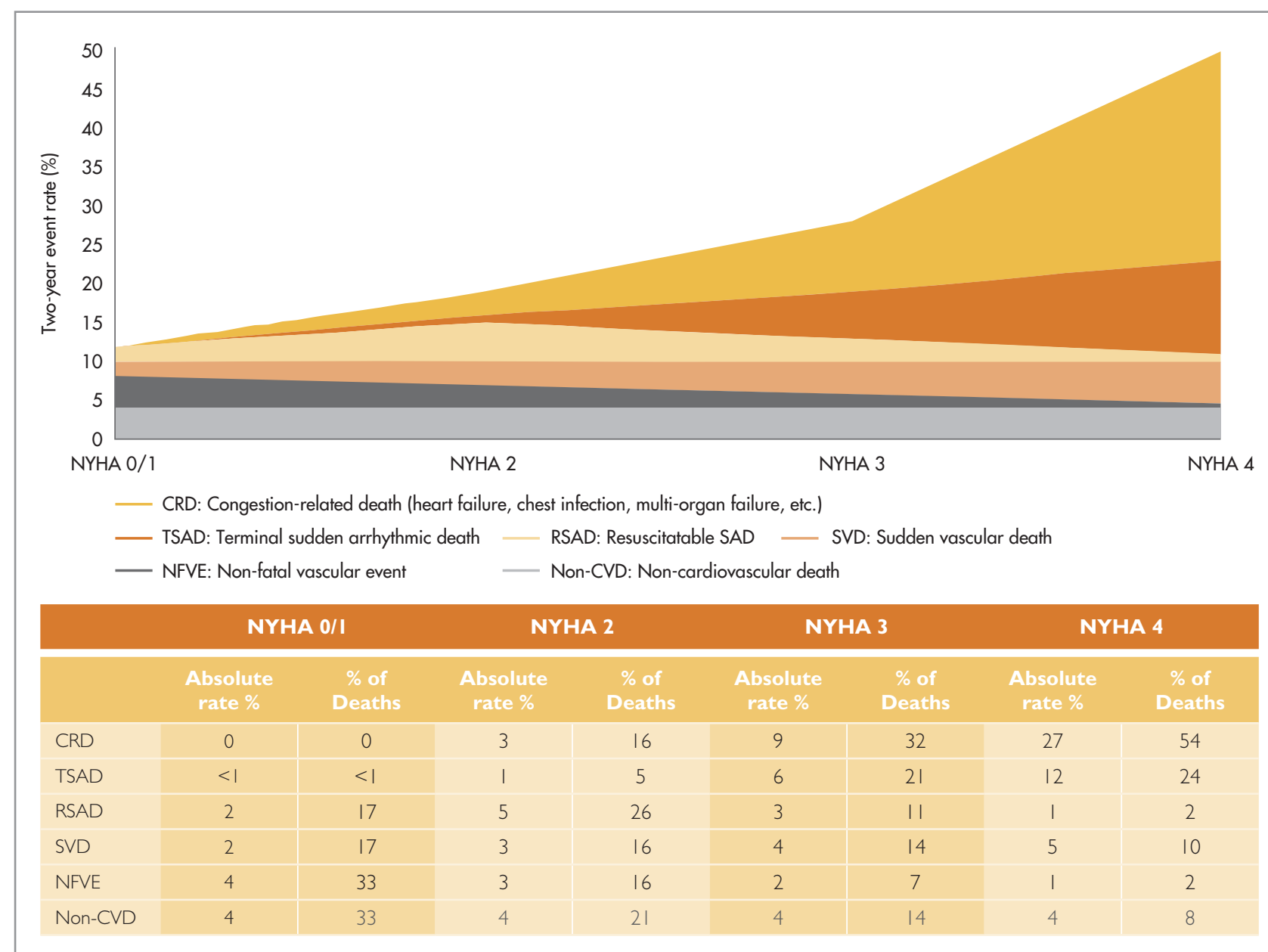

FIGURE 5: Two-year cause-specific mortality and non-fatal vascular events for patients with cardiovascular disease according to New York Heart Association (NYHA) class.

Numbers and proportions are a conceptual representation of absolute and relative risk and are not strictly evidence-based. Note that for patients in New York Heart Association Class 4, interventions for sudden arrhythmic death may be ineffective or fail to lead to a meaningful prolongation of life because the patient is likely to die soon of worsening heart failure.

$C R D=$ congestion-related death, otherwise called death due to worsening heart failure, NFVE = non-fatal vascular event (e.g. myocardial infarction and stroke; note that events are more likely to be suddenly fatal as heart failure progresses), Non-CVD = non-cardiovascular death, RSAD = resuscitatable sudden arrhythmic death, SVD = sudden vascular death, TSAD = terminal (non-resucitatable) sudden arrhythmic death. ${ }^{(78)}$ 
combined with programmed ventricular stimulation. ${ }^{(83)}$ The algorithm yielded an excellent sensitivity and negative predictive value (arguably the most important parameter) of 100\%, as well as a specificity of $93.8 \%$; on the downside, positive predictive value was only $22 \%$. Modern imaging modalities such as MRI may further yield added value in identifying patients at increased risk of ventricular arrhythmias who may benefit from ICD implantation. ${ }^{\left({ }^{4}\right)}$ Similar algorithms are being developed also for rarer disease entities such as arrhythmogenic right ventricular cardiomyopathy (ARVC). (74) If proven positive in randomised clinical outcome trials, these concepts may move the field closer to venturing beyond the current (suboptimal) standard of LVEF for risk stratification. Until such outcome trials are available, however, it may be prudent to stick to the currently available evidence and guideline recommendations; at the same time, recruitment into ongoing trials is encouraged in order to accelerate the generation of high-level evidence that may potentially alter current clinical practice.

Cardiac resynchronisation therapy remains an important treatment modality for heart failure patients to induce reverse LV remodelling and to improve morbidity and mortality. However, the rate of so-called "non-responders" remains in the order of $20 \%$ - 30\%, depending on definitions and cut-offs. ${ }^{(85)}$ The MORE-CRT MPP trial investigated the effect of stimulating the LV from 2 sites instead of 1 to reduce the number of nonresponders. ${ }^{(86)}$ Five hundred and forty-four patients classified as non-responders (defined as an LV end-systolic volume reduction by <15\%) 6 months after CRT implantation were randomised to receive the "Multipoint"'TM algorithm turned on (MPP ON) or off (standard of care group). While the conversion rate to "responders" was no different between the 2 groups (31.8\% vs. $33.8 \%$ ), patients in the MPP group programmed to a wide electrode distance were significantly more likely to convert to responders than those programmed to other vector combinations (45.6\% vs. $26.2 \%, p=0.006$ ). ${ }^{(86)}$ Although interesting and biologically plausible, these findings have to be viewed as hypothesis-generating in view of the negative primary endpoint.

Conflict of interest: A.J.C. has received personal fees and institutional grants from Bayer, Boehringer Ingelheim, Daiichi Sankyo and BMS/ Pfizer, and personal fees from Medtronic, Boston Scientific and Abbott. Professor Lip is a consultant for Bayer/Janssen, BMS/Pfizer, Medtronic, Boehringer Ingelheim, Novartis, Verseon and Daiichi-Sankyo, and a speaker for Bayer, BMS/Pfizer, Medtronic, Boehringer Ingelheim, and DaiichiSankyo. No fees are directly received personally. R.S. reports other income from Rhythm Al, grants, personal fees and nonfinancial support from Medtronic, grants, personal fees and non-financial support from Biosense Webster, personal fees and non-financial support from Abbott, personal fees and non- financial support from Boston Scientific, during the conduct of the study; in addition personal fees and non-financial support from Daiichi Sankyo, non-financial support from Boeringher Ingleheim, outside the submitted work. In addition, Dr Schilling has a patent Rhythm Al - STAR mapping pending. J.S. has received consultant and/or speaker fees from Abbott, Amgen, Astra-Zeneca, Atricure, Bayer, Biosense Webster, Biotronik, Boehringer-Ingelheim, Boston Scientific, Bristol-Myers Squibb, Daiichi Sankyo, Medscape, Medtronic, Merck/MSD, Novartis, Pfizer, Sanofi-Aventis, WebMD, and Zoll. He reports ownership of CorXL. H.C. reports personal fees from Abbott Medical, personal fees from Atricure, personal fees from Biosense Webster, personal fees from Boston Scientific, personal fees from Medtronic, outside the submitted work. J.S. has received grant support through his institution from Abbott, Bayer Healthcare, Biosense Webster, Biotronik, Boston Scientific, Daiichi Sankyo, and Medtronic.

\section{REFERENCES}

I. Chen Q, Xu J, Gianni C, et al. Simple electrocardiographic criteria for rapid identification of wide QRS complex tachycardia: The new limb lead algorithm. Heart Rhythm 2019;5271.

2. May AM, DeSimone CV, Kashou AH, et al. The WCT formula: A novel algorithm designed to automatically differentiate wide-complex tachycardias. J Electrocardiol 2019;54:61-68.

3. May AM, Brenes-Salazar JA, DeSimone CV, et al. Electrocardiogram algorithms used to differentiate wide complex tachycardias demonstrate diagnostic limitations when applied by non-cardiologists. J Electrocardiol 2018:51:1103-1109.

4. Pachón M, Arias MA, Salvador-Montañés Ó, et al. A scoring algorithm for the accurate differential diagnosis of regular wide QRS complex tachycardia. Pacing Clin Electrophysiol 2019:42:625-633.

5. Hwang J, Kim J, Choi KJ, et al. Assessing accuracy of wrist-worn wearable devices in measurement of paroxysmal supraventricular tachycardia heart rate. Korean Circ J 2019;49:437-445.

6. Ernst S, Cazzoli I, Guarguagli S. An initial experience of high-density mappingguided ablation in a cohort of patients with adult congenital heart disease. Europace 2019;21:i43-i53.

7. Raja JM, Cave B, Jefferies JL, et al. Etripamil: Intranasal calcium channel blocker: A novel noninvasive modality in the treatment of paroxysmal supraventricular tachycardia. Curr Probl Cardiol 2019; S0I46-2806(19)30 I03-3.

8. Faisaluddin M, Ashish K, Hajra A, et al. Etripamil: Self-management of supraventricular tachycardia is not far away? Int J Cardiol Heart Vasc 2019:22:82-83.

9. Hu J, Yu J, Chen Q, et al. Efficacy of nifekalant in patients with WolffParkinson-White syndrome and atrial fibrillation: Electrophysiological and clinical findings. J Am Heart Assoc 2019:8:e01251।.

10. Brugada J, Katritsis DG, Arbelo E, et al.; ESC Scientific Document Group. 2019 ESC Guidelines for the management of patients with supraventricular tachycardia. The Task Force for the management of patients with supraventricular tachycardia of the European Society of Cardiology (ESC). Eur Heart J 2019; pii:ehz467. doi:10.1093/eurheartj/ehz467.

II. Allan V, Honarbakhsh S, Casas JP, et al. Are cardiovascular risk factors also associated with the incidence of atrial fibrillation? A systematic review and field synopsis of 23 factors in 32 population-based cohorts of 20 million participants. Thromb Haemost 2017;1 17:837-850. 


\section{REFERENCES}

12. Lim YM, Yang PS, Jang E, et al. Body mass index variability and long-term risk of new-onset atrial fibrillation in the general population: A Korean Nationwide Cohort Study. Mayo Clin Proc 2019;94:225-235.

13. Linz D, Brooks AG, Elliott AD, et al. Variability of sleep apnea severity and risk of atrial fibrillation: The VARIOSA-AF study. JACC Clin Electrophysiol 2019:5:692-701.

14. Li YG, Pastori D, Farcomeni A, et al. A Simple Clinical Risk Score (C2HEST) for predicting incident atrial fibrillation in Asian subjects: Derivation in 47| 446 Chinese subjects, with internal validation and external application in 45। 199 Korean subjects. Chest 2019;155:510-518.

15. Li YG, Bisson A, Bodin A, et al. C2HEST Score and prediction of incident atrial fibrillation in poststroke patients: A French Nationwide Study. J Am Heart Assoc 2019;8:e0 2546.

16. Hart RG, Sharma M, Mundl H, et al. Rivaroxaban for stroke prevention after embolic stroke of undetermined source. N Engl J Med 2018;378:2191-2201.

17. Diener $H C$, Sacco RL, Easton JD, et al. Dabigatran for prevention of stroke after embolic stroke of undetermined source. N Engl J Med 2019; 380:1906-1917.

18. Mairesse GH, Moran P, Van Gelder IC, et al. Screening for atrial fibrillation: A European Heart Rhythm Association (EHRA) consensus document endorsed by the Heart Rhythm Society (HRS), Asia Pacific Heart Rhythm Society (APHRS), and Sociedad Latinoamericana de Estimulacion Cardiaca y Electrofisiologia (SOLAECE). Europace 2017;19:1589-1623.

19. Perez MV, Mahaffey KW, Hedlin H, et al.; Apple Heart Study Investigators. Large-scale assessment of a smartwatch to identify atrial fibrillation. N Engl J Med 2019;381:1909-1917

20. Guo Y, Lane DA, Wang L, et al.; mAF-App II Trial Investigators. Mobile Health (mHealth) technology for improved screening, patient involvement and optimising integrated care in atrial fibrillation: The mAFA (mAF-App) ॥ randomised trial. Int J Clin Pract 2019;73:el 3352.

21. Jung $H$, Yang PS, Sung JH, et al. Hypertrophic cardiomyopathy in patients with atrial fibrillation: Prevalence and associated stroke risks in a Nationwide Cohort Study. Thromb Haemost 2019;1 19:285-293.

22. Steensig K, Olesen KKW, Thim T, et al. Should the presence or extent of coronary artery disease be quantified in the $\mathrm{CHA}_{2} \mathrm{DS}_{2}-\mathrm{VASc}$ score in atrial fibrillation? A report from the Western Denmark Heart Registry. Thromb Haemost 2018;1 18:2162-2170

23. Attia Zl, Noseworthy PA, Lopez-Jimenez F, et al. An artificial intelligenceenabled ECG algorithm for the identification of patients with atrial fibrillation during sinus rhythm: A retrospective analysis of outcome prediction. Lancet 2019;394:861-867.

24. Borre ED, Goode A, Raitz G, et al. Predicting thromboembolic and bleeding event risk in patients with non-valvular atrial fibrillation: A systematic review. Thromb Haemost 2018; 1 8:2171-2187.

25. Chao TF, Lip GYH, Lin YJ, et al. Incident risk factors and major bleeding in patients with atrial fibrillation treated with oral anticoagulants: A comparison of baseline, follow-up and delta HAS-BLED scores with an approach focused on modifiable bleeding risk factors. Thromb Haemost 20 I 8; I I 8:768-777.

26. Guo $Y, Z$ hu H, Chen $Y$, et al. Comparing bleeding risk assessment focused on modifiable risk factors only versus validated bleeding risk scores in atrial fibrillation. Am J Med 2018;131:185-192.

27. Rivera-Caravaca JM, Marín F, Vilchez JA, et al. Refining stroke and bleeding prediction in atrial fibrillation by adding consecutive biomarkers to clinical risk scores. Stroke 2019;50:1372-1379.

28. Chang TY, Lip GYH, Chen SA, et al. Importance of risk reassessment in patients with atrial fibrillation in guidelines: Assessing risk as a dynamic process. Can J Cardiol 2019;35:61|-6/8.

29. Chao TF, Liao JN, Tuan TC, et al. Incident co-morbidities in patients with atrial fibrillation initially with a $\mathrm{CHA}_{2} \mathrm{DS}_{2}$-VASc Score of 0 (Males) or I (Females): Implications for reassessment of stroke risk in initially "low-risk" patients. Thromb Haemost 2019;1 19:1 162-1170.
30. Fanaroff AC, Steffel J, Alexander JH, et al. Stroke prevention in atrial fibrillation: Re-defining "real-world data" within the broader data universe. Eur Heart J 2018;39:2932-2941.

31. Lee SR, Choi EK, Han KD, et al. Edoxaban in Asian patients with atrial fibrillation: Effectiveness and safety. J Am Coll Cardiol 20 18;72:838-853.

32. Deitelzweig S, Keshishian A, Li X, et al. Comparisons between oral anticoagulants among older nonvalvular atrial fibrillation patients. J Am Geriatr Soc 2019;67:1662-1671.

33. Chao TF, Liu CJ, Lin YJ, et al. Oral anticoagulation in very elderly patients with atrial fibrillation: A Nationwide Cohort Study. Circulation 20 18;138:37-47.

34. Paquette $M$, Riou França $L$, Teutsch $C$, et al. Persistence with dabigatran therapy at 2 years in patients with atrial fibrillation. J Am Coll Cardiol 2017; 70:1573-1583.

35. Montalescot G, Brotons C, Cosyns B, et al. Educational impact on apixaban adherence in atrial fibrillation (the AEGEAN STUDY): A randomised clinical trial. Am J Cardiovasc Drugs 2019;doi: 10.1007/s40256-019-00356-2.

36. Lee SR, Choi EK, Han KD, et al. Non-vitamin K antagonist oral anticoagulants in Asian patients with supranormal renal function. Stroke 2019; 50:1480-1489.

37. Siontis KC, Zhang $X$, Eckard A, et al. Outcomes associated with apixaban use in patients with end-stage kidney disease and atrial fibrillation in the United States. Circulation 2018;138:1519-1529.

38. Hohnloser SH, Camm J, Cappato R, et al. Uninterrupted administration of edoxaban vs vitamin $\mathrm{K}$ antagonists in patients undergoing atrial fibrillation catheter ablation: Rationale and design of the ELIMINATE-AF study. Clin Cardiol 2018;41:440-449.

39. Calkins H, Willems S, Gerstenfeld EP, et al. Uninterrupted dabigatran versus warfarin for ablation in atrial fibrillation. N Engl J Med 2017;376: 1627-I 636.

40. Ezekowitz MD, Pollack CVJr, Halperin JL, et al. Apixaban compared to heparin/vitamin K antagonist in patients with atrial fibrillation scheduled for cardioversion: The EMANATE trial. Eur Heart J 2018;39:2959-297।.

41. Vranckx P, Valgimigli M, Eckardt $L$, et al. Edoxaban-based versus vitamin $K$ antagonist-based antithrombotic regimen after successful coronary stenting in patients with atrial fibrillation (ENTRUST-AF PCI): A randomised, openlabel, phase 3b trial. Lancet 2019;394:1335-1343.

42. Lopes RD, Heizer G, Aronson R, et al. Antithrombotic therapy after acute coronary syndrome or $\mathrm{PCl}$ in atrial fibrillation. $\mathrm{N}$ Engl J Med 2019; 380: 1509- 1524.

43. Lopes RD, Hong H, Harskamp RE, et al. Safety and efficacy of antithrombotic strategies in patients with atrial fibrillation undergoing percutaneous coronary intervention: A network meta-analysis of randomised controlled trials. JAMA Cardiol 2019;4:747.

44. Yasuda S, Kaikita K, Akao M, et al. Antithrombotic therapy for atrial fibrillation with stable coronary disease. N Engl J Med 20 19;38 I: I I03-1 I I 3.

45. Lip G. The ABC pathway: An integrated approach to improve AF management. Nat Rev Cardiol 2017; 14:627-628.

46. Gallagher C, Elliott AD, Wong CX, et al. Integrated care in atrial fibrillation: A systematic review and meta-analysis. Heart 2017; 103:1947- 1953.

47. Yoon M, Yang PS, Jang E, et al. Improved population-based clinical outcomes of patients with atrial fibrillation by compliance with the simple $A B C$ (Atrial Fibrillation Better Care) pathway for integrated care management: A Nationwide cohort study. Thromb Haemost 2019;19:1695-1703.

48. Pastori D, Pignatelli P, Menichelli D, et al. Integrated care management of patients with atrial fibrillation and risk of cardiovascular events: The $A B C$ (Atrial fibrillation Better Care) pathway in the ATHERO-AF study cohort. Mayo Clin Proc 2019;94:1261-1267.

49. Pastori D, Farcomeni A, Pignatelli P, et al. ABC (Atrial fibrillation Better Care) pathway and healthcare costs in atrial fibrillation: The ATHERO-AF study. Am J Med 2019; | 32:856-86|. 
50. Proietti M, Romiti GF, Olshansky B, et al. Improved outcomes by integrated care of anticoagulated patients with atrial fibrillation using the simple $A B C$ (Atrial Fibrillation Better Care) pathway. Am J Med 20 I 8; I 31:1359-1366.e6.

51. Packer DL, Mark DB, Robb RA, et al.; CABANA Investigators. Effect of catheter ablation vs antiarrhythmic drug therapy on mortality, stroke, bleeding, and cardiac arrest among patients with atrial fibrillation. The CABANA Randomised Clinical Trial. JAMA 2019;321:1261 - 274.

52. Jin MN, Kim TH, Kang KW, et al. Atrial fibrillation catheter ablation improves I-year follow-up cognitive function, especially in patients with impaired cognitive function. Circ Arrhythm Electrophysiol 2019;12:e007197.

53. Blomström-Lundqvist C, Gizurarson S, Schwieler J, et al. Effect of catheter ablation vs antiarrhythmic medication on quality of life in patients with atrial fibrillation. The CAPTAF Randomised Clinical Trial. JAMA 2019; 321:1059-1068.

54. Mörtsell D, Arbelo E, Dagres N, et al.; ESC-EHRA Atrial Fibrillation Ablation Long-Term Registry Investigators. Cryoballoon vs. radiofrequency ablation for atrial fibrillation: A study of outcome and safety based on the ESC-EHRA atrial fibrillation ablation long-term registry and the Swedish catheter ablation registry. Europace 2019;21:581-589.

55. Bollmann A, Ueberham L, Schuler E, et al. Cardiac tamponade in catheter ablation of atrial fibrillation: German-wide analysis of 21 | $4 \mid$ procedures in the Helios atrial fibrillation ablation registry (SAFER). Europace 2018; 20:1944-1951.

56. Hoffmann E, Straube F, Wegscheider K, et al.; FREEZE Cohort Study Investigators. Outcomes of cryoballoon or radiofrequency ablation in symptomatic paroxysmal or persistent atrial fibrillation. Europace 2019; 21:1313-1324.

57. Landolina M, Arena G, lacopino S, et al. Center experience does not influence long-term outcome and peri-procedural complications after cryoballoon ablation of paroxysmal atrial fibrillation: Data on 860 patients from the real-world multicenter observational project. Int J Cardiol 20 I8; 272:130-136.

58. Holmqvist F, Kesek M, Englund A, et al. A decade of catheter ablation of cardiac arrhythmias in Sweden: Ablation practices and outcomes. Eur Heart J 2019:40:820-830.

59. Grecu M, Blomström-Lundqvist C, Kautzner J, et al. In-hospital and I2-month follow-up outcome from the ESC-EORP EHRA atrial fibrillation ablation long-term registry: Sex differences. Europace 2019; doi: 10.1093/europace/ euz225.

60. Giehm-Reese M, Kronborg MB, Lukac P, et al. Recurrent atrial flutter ablation and incidence of atrial fibrillation ablation after first-time ablation for typical atrial flutter: A nation-wide Danish cohort study. Int J Cardiol 2019;19:33183.

61. Komatsu Y, Hocini M, Nogami A, et al. Catheter ablation of refractory ventricular fibrillation storm after myocardial infarction: A multicenter study. Circulation 2019;1 39:2315-2325.

62. Mahida S, Venlet J, Saguner AM, et al. Ablation compared with drug therapy for recurrent ventricular tachycardia in arrhythmogenic right ventricular cardiomyopathy: Results from a multicenter study. Heart Rhythm 2019; 16:536-543.

63. Winkle RA, Mohanty S, Patrawala RA, et al. Low complication rates using high power $(45-50 \mathrm{~W})$ for short duration for atrial fibrillation ablations. Heart Rhythm 2019;16:165-169.

64. Melby DP, Gornick C, Abdelhadi R, et al. Outcomes following persistent atrial fibrillation ablation using localised sources identified with Ripple map. J Cardiovasc Electrophysiol 2019;30:1860-1867.

65. Luther V. Agarwal S, Chow A, et al.; Kanagaratnam PRipple-AT Study. A multicenter and randomised study comparing 3D mapping techniques during atrial tachycardia ablations. Circ Arrhythm Electrophysiol 20 I 9; I 2:e007394.

66. Xie S, Kubala M, Liang JJ, et al. Utility of ripple mapping for identification of slow conduction channels during ventricular tachycardia ablation in the setting of arrhythmogenic right ventricular cardiomyopathy. J Cardiovasc Electrophysiol 2019;30:366-373.
67. Willems S, Verma A, Betts TR, et al. Targeting nonpulmonary vein sources in persistent atrial fibrillation identified by noncontact charge density mapping UNCOVER AF Trial. Circ Arrhythm Electrophysiol 20 I 9; I :e007233.

68. Honarbakhsh S, Hunter RJ, Ullah W, et al. Ablation in persistent atrial fibrillation using stochastic trajectory analysis of ranked signals (STAR) mapping method. JACC Clin Electrophysiol 2019;5:817-829.

69. Reddy VY, Neuzil P, Koruth JS, et al. Pulsed field ablation for pulmonary vein isolation in atrial fibrillation. J Am Coll Cardiol 2019;74:315-326.

70. Robinson CG, Samson PP, Moore KMS, et al. Phase 1/II Trial of electrophysiology-guided noninvasive cardiac radioablation for ventricular tachycardia. Circulation 2019;139:313-321.

7I. Cronin EM, Bogun FM, Maury P, et al. 2019 HRS/EHRA/APHRS/LAHRS expert consensus statement on catheter ablation of ventricular arrhythmias: Executive summary. Europace;doi: 10.1093/europace/euzl 32

72. Linde C, ESC Scientific Document Group, Bongiorni MG, et al. Sex differences in cardiac arrhythmia: A consensus document of the European Heart Rhythm Association, endorsed by the Heart Rhythm Society and Asia Pacific Heart Rhythm Society. Europace 20 18;20:1565-1565ao.

73. Towbin JA, McKenna WJ, Abrams DJ, et al. 2019 HRS expert consensus statement on evaluation, risk stratification, and management of arrhythmogenic cardiomyopathy. Heart Rhythm 2019; 16:e301-e372.

74. Cadrin-Tourigny J, Bosman LP, Nozza A, et al. A new prediction model for ventricular arrhythmias in arrhythmogenic right ventricular cardiomyopathy. Eur Heart J 2019:40:1850-1858.

75. Chatterjee D, Fatah M, Akdis D, et al. An autoantibody identifies arrhythmogenic right ventricular cardiomyopathy and participates in its pathogenesis. Eur Heart J 20 I 8;39:3932-3944.

76. Sondergaard KB, Wissenberg M, Gerds TA, et al. Bystander cardiopulmonary resuscitation and long-term outcomes in out-of-hospital cardiac arrest according to location of arrest. Eur Heart J 2019;40:309-318.

77. Cleland JGF, Hindricks G, Petrie M. The shocking lack of evidence for implantable cardioverter defibrillators for heart failure; with or without cardiac resynchronisation. Eur Heart J 2019;40:2128-2130.

78. Barra S, Duehmke R, Providencia R, et al. Very long-term survival and late sudden cardiac death in cardiac resynchronisation therapy patients. Eur Heart J 2019:40:2121-2127.

79. Packer $M$. What causes sudden death in patients with chronic heart failure and a reduced ejection fraction? Eur Heart J 2019; doi: 10.1093/eurheartj/ ehz553.

80. Steffel J. The subcutaneous implantable cardioverter defibrillator. Eur Heart J 2017;38:226-228.

81. Galand V, Polin B, Martins RP, et al. An entirely leadless cardiac resynchronisation therapy. Eur Heart J 2019;40:858-859.

82. Olsen T, Jorgensen OD, Nielsen JC, et al. Incidence of device-related infection in 97750 patients: Clinical data from the complete Danish devicecohort (1982 - 2018). Eur Heart J 2019;40:1862-1869.

83. Gatzoulis KA, Tsiachris D, Arsenos P, et al. Arrhythmic risk stratification in post-myocardial infarction patients with preserved ejection fraction: The PRESERVE EF study. Eur Heart J 2019;40:2940-2949.

84. Gutman SJ, Costello BT, Papapostolou S, et al. Reduction in mortality from implantable cardioverter-defibrillators in non-ischaemic cardiomyopathy patients is dependent on the presence of left ventricular scar. Eur Heart J 2019;40:542-550.

85. Steffel J, Ruschitzka F. Super response to cardiac resynchronisation therapy. Circulation 2014;130:87-90.

86. Leclercq C, Burri H, Curnis A, et al. Cardiac resynchronisation therapy nonresponder to responder conversion rate in the more response to cardiac resynchronisation therapy with MultiPoint Pacing (MORE-CRT MPP) study: Results from Phase I. Eur Heart J 2019;40:2979-2987. 